\title{
Asociación entre variables psicológicas y sociales con la adhesión en personas con diabetes tipo 2
}

\section{Association between psychological and social variables with adherence among people with type 2 diabetes}

\author{
Angélica Magali Torres y Julio Alfonso Piña* \\ Investigadores Independientes Hermosillo, México
}

(Rec: 09 mayo 2009 / Acep: 29 abril 2010)

\begin{abstract}
Resumen
Se presentan los resultados de un estudio transversal que contó con la participación de 334 personas adultas con diagnóstico de diabetes tipo 2 de la ciudad de Hermosillo, México, cuyo objetivo fue evaluar la asociación entre variables psicológicas y sociales con tres comportamientos de adhesión: ejercicio físico, dieta y consumo de medicamentos. Un análisis de regresión logística multivariada reveló asociaciones de éstos con bajos niveles de estrés vinculado con toma de decisiones y tolerancia a la frustración, así como con apoyo social percibido. Los resultados sugieren el peso diferenciado que esas variables tienen sobre tres comportamientos de adhesión a la terapéutica en personas con diagnóstico de diabetes tipo 2, aspecto que debe ser tenido en cuenta al momento de diseñar e instrumentar programas de intervención interdisciplinarias dirigidos a mejorar la práctica consistente y eficiente de los mencionados comportamientos.

Palabras Clave: Diabetes, adhesión, estrés, apoyo social, programas de intervención.
\end{abstract}

\begin{abstract}
We present the results of a cross-sectional study in which participated 334 adult persons with diagnosis of type 2 diabetes at Hermosillo city, Mexico, with the main objective to assess the association between psychological and social variables with three adherence behaviors: physical exercise, diet, and medication consumption. A logistic regression analysis showed several associations for the analyzed behaviors with low stress-related to decision-making and tolerance to frustration, as well as with perceived social support The results of this study suggest a differential weight of these variables on adherence behaviors in persons with diagnosis of type 2 diabetes, aspect that must taken into account at time of designing and implementing interdisciplinary intervention programs directed to promote the consistently and efficiently practice of these behaviors.
\end{abstract}

Key Words: Diabetes, adherence, stress, social support, intervention programs. 


\section{Introducción}

En México, la diabetes tipo 2 ha alcanzado proporciones epidémicas. En efecto, sirva como ejemplo mencionar que en el periodo 2000-2004 las tasas de morbilidad por cada 100 mil habitantes se incrementaron poco más de $70 \%$, pasando de 287.2 casos en el primero de esos años a 384.0 en el último (Secretaría de Salud Pública, 2006); asimismo, en lo que va de la actual década se ha ubicado junto con la cardiopatía isquémica como una de las tres principales causas de muerte en personas adultas (Córdova-Villalobos et al., 2008).

Partiendo del entendido de que la diabetes es una enfermedad crónica que resulta excesivamente costosa tanto para las instituciones del sector salud de nuestro país como para las personas que la padecen y sus familias, ${ }^{1}$ en las actuales circunstancias se ha vuelto una prioridad el diseño, instrumentación y evaluación de programas de intervención interdisciplinaria dirigidos a reducir esos costos a través de dos vías: primera, previniendo su aparición en los sectores de la población especialmente vulnerables - i.e., personas obesas, con sobrepeso o con antecedentes familiares de diabetes-; segunda, promoviendo la práctica eficiente de los comportamientos de adhesión que son necesarios en razón de la enfermedad, mismos que incluyen la realización de ejercicio físico dosificado, la adopción de una dieta, el monitoreo permanente de los niveles de glucosa en sangre y el consumo de medicamentos o la inyección de insulina.

Por lo que hace a esta última, en una considerable cantidad de estudios se ha establecido que diferentes variables se asocian con o predicen la práctica de los mencionados comportamientos; destacan, por un lado, la edad, el género y el nivel educativo (Babwah et al., 2006); por el otro, el locus de control, la autoeficacia y experimentar bajos niveles de depresión y estrés (Fisher et al., 2007; O'Hea et al., 2009); finalmente, contar con redes de apoyo social en sus modalidades informativo y emocional (Brownson \& Heisler, 2009; Tang, Brown, Funnell \& Anderson, 2008), principalmente.

Ciertamente, llama nuestra atención que en la mayoría de los casos se trata de estudios conducidos sin el apoyo en modelos teóricos articulados en lo conceptual, cuya utilidad quedaría demostrada en la medida en que se cumplieran con al menos tres condiciones: primera, la definición clara e incontrovertida de las variables con las que se presume se está haciendo referencia a lo psicológico; segunda, que permitieran comprender por qué y en qué circunstancias las personas practican o no los comportamientos de adhesión, y finalmente, que a su vez permitieran traducir los hallazgos en propuestas viables de intervención susceptibles de

\footnotetext{
Se calcula que en el año 2000 los costos de la diabetes en México ascendieron a más de 15 mil millones de dólares, aun cuando tan sólo 1974 millones se utilizaron como costos directos para su tratamiento (Barceló, Aedo, Rajpathak \& Robles, 2003).
}

ser evaluadas cuantas veces fuera necesario (Piña, Ybarra, Alcalá \& Samaniego, en prensa).

Sobre la base de esta premisa es que recientemente nos dimos a la tarea de elaborar un modelo psicológico para la investigación de los comportamientos de adhesión terapéutica en personas que viven con el Virus de la Inmunodeficiencia Humana - VIH (Piña \& Sánchez-Sosa, 2007), el cual un año después fue adaptado con éxito al problema de la diabetes tipo 2 (Piña \& Torres, 2008). En dicho modelo se parte del supuesto de que la práctica eficiente de los comportamientos de adhesión se puede ver afectada por distintas variables psicológicas de tipo histórico - situaciones vinculadas con estrés, motivación y competencias conductuales - y/o del presente - comportamientos asociados a la enfermedad (Figura 1), así como por otras de naturaleza biológica y social.

Tal y como se observa en ésta, en la fase uno adquieren especial relevancia tres variables psicológicas de tipo histórico. Las situaciones vinculadas con estrés, que se relacionan con el fenómeno de personalidad y que definidas aquí como campos de contingencias que se caracterizan porque es el comportamiento de las personas el que regula diferencialmente las propiedades funcionales de los agentes del ambiente en su acción sobre el organismo; son situaciones en las que: a) las consecuencias al comportarse son impredictibles y opcionales —impredictibilidad-; b) las señales o eventos de estímulo a las que hay que responder son ambiguas — ambigüedad-, y c) las consecuencias de estímulo al responder, nocivas biológicamente, o su pérdida, son independientes del comportamiento de las personas -incertidumbre (Ribes, 1990).

Por lo que hace a las competencias conductuales, éstas tienen que ver con la funcionalidad adquirida en el pasado, misma que permite a una persona interactuar de manera eficiente en situaciones en donde se prescriben ciertos criterios; ser competente equivale a decir que una persona dispone de los recursos - conocimiento, habilidades y destrezas - para poner en práctica los comportamientos de adhesión: se sabe qué es la diabetes y las potenciales complicaciones; se sabe en qué consiste el tratamiento y por qué se le debe seguir puntualmente, y adicionalmente, se hacen las cosas que se espera: se realiza ejercicio físico dosificado, se adopta una dieta balanceada, se monitorean los niveles de glucosa y se consumen los medicamentos prescritos por el personal de salud.

El último elemento de la fase uno es el de motivos. Es una variable que pertenece a las categorías disposicionales de inclinaciones o propensiones, que se define en términos de querer hacer algo en situaciones socialmente valoradas. Cuando se dice que una persona se encuentra motivada para comportarse de una manera u otra, significa que se inclina o es propensa a hacer algo, siempre y cuando lo quiera hacer porque lo considera oportuno y pertinente; en tal sentido, una persona elige u opta practicar determinado 
Fig. 1. Representación gráfica del modelo psicológico para la investigación de los comportamientos de adhesión (adaptado a la diabetes tipo 2).

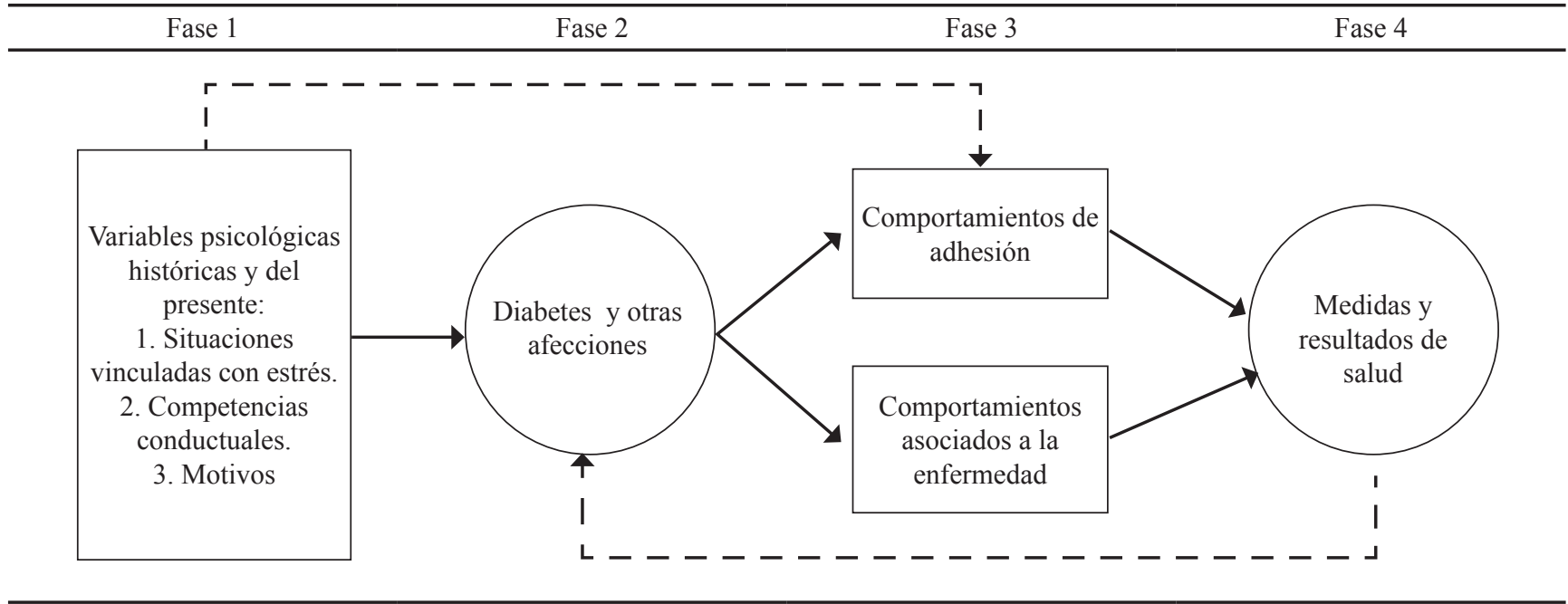

comportamiento a partir de la obtención de consecuencias de estímulo positivas, lo que hará más probable que ese comportamiento se siga practicando en el futuro en situaciones funcionalmente similares (Piña, 2009; Ribes, 2005).

Una vez diagnosticada la enfermedad - fase dos- y que la persona con diabetes ha iniciado con un plan de intervención, se espera que practique los comportamientos de adhesión - fase tres_- En caso de hacerlo en los términos planteados por el personal de salud, lo que se tendría en la fase cuatro no sería otra cosa que mantener estable la funcionalidad biológica — bajos niveles de hemoglobina glucosilada y glucosa, principalmente-; en caso contrario, es probable que en el corto o mediano plazos aparezcan otras afecciones propias de la fase dos, como por ejemplo hipertensión arterial, cardiopatía isquémica, hiperlipidemia e hiperglucemia, por mencionar unas cuantas (Rae, 2006). En la misma fase tres se encuentran los comportamientos asociados a la enfermedad, que corresponden a una diversidad de comportamientos que correlacionan con la presencia de una patología biológica en tres posible direcciones: a) se pueden presentar los comportamientos como el efecto de la patología; b) hay comportamientos que derivan de la terapéutica que se aplica — como por ejemplo el vómito y la náusea producidos por la quimioterapia en padecimientos oncológicos y por lo medicamentos antirretrovirales en la infección por el VIHy c) los comportamientos que se vinculan indirectamente con la patología, tales como ansiedad, depresión, tensión, desesperación, irritabilidad, ira, etcétera.

Teniendo en cuenta algunos de los elementos del modelo, en el presente trabajo se planteó la pregunta de investigación de si la práctica o no de los comportamientos de adhesión se vería afectada de manera diferencial por un conjunto de variables psicológicas -i.e., situaciones vinculadas con estrés - y sociales - i.e., apoyo socialPor tanto, el estudio se condujo para dar cumplimiento al objetivo de identificar cuáles de esas variables se asociarían con tres comportamientos de adhesión en personas con diagnóstico de diabetes tipo 2.

\section{Método}

\section{Participantes}

El presente estudio de corte transversal se llevó al cabo en la ciudad de Hermosillo, México. La selección de los participantes se realizó con base en un muestreo por disponibilidad de derechohabientes del Instituto Mexicano del Seguro Social _-IMSS - y dos asociaciones civiles para personas con diabetes de la localidad. En el caso de los primeros se consideraron a quienes acudieron a las citas programadas con el médico especialista responsable del tratamiento; en el caso de los segundos a quienes cada semana asistieron a sesiones grupales donde se abordaban asuntos de interés colectiva. Como criterios de inclusión se consideraron los siguientes: a) tener diagnóstico de diabetes tipo 2 mayor de tres meses; b) tener una edad entre 18 y 70 años; c) encontrarse expuesto a un régimen de tratamiento con hipoglucemiantes orales o inyección de insulina. De una muestra original de 402 participantes se eliminaron 68 porque no cumplieron con alguno de esos criterios, por lo que la muestra definitiva quedó conformada por 334 personas con diabetes tipo 2, con una edad mediana de 55 años $(\mathrm{DE}=10.09)$ y edades mínima y máxima de 22 y 70 años, respectivamente; asimismo, $113(33.8 \%)$ eran hombres y 221 (66.2\%) mujeres; las restantes características sociodemográficas y clínicas se resumen en la Tabla 1.

\section{Procedimiento}

El protocolo de investigación fue previamente revisado y aprobado por los Comités de Investigación y Ética del 
Tabla 1. Características sociodemográficas y clínicas de los participantes.

\begin{tabular}{|c|c|c|c|c|c|c|}
\hline \multicolumn{2}{|c|}{ Variables } & Mediana & $\mathrm{DE}$ & Rango & $\mathrm{N}$ & $\%$ \\
\hline \multicolumn{2}{|l|}{ Edad } & 55 & 10.09 & $22-70$ & & \\
\hline Sexo & $\begin{array}{l}\text { Masculino } \\
\text { Femenino }\end{array}$ & & & & $\begin{array}{l}113 \\
221\end{array}$ & $\begin{array}{l}33.8 \\
66.2\end{array}$ \\
\hline \multicolumn{2}{|c|}{ Origen } & & & & 165 & 49.4 \\
\hline & IMSS (HRZ-2) & & & & 82 & 24.6 \\
\hline & IMSS (UMF-38) & & & & 87 & 26.0 \\
\hline & Asociaciones civiles & & & & 87 & 26.0 \\
\hline \multicolumn{7}{|c|}{ Estado Civil } \\
\hline & Solteras (os) & & & & 11 & 3.3 \\
\hline & Casadas (os) & & & & 230 & 68.9 \\
\hline & Unión Libre & & & & 14 & 4.2 \\
\hline & Separadas (os) & & & & 25 & 7.5 \\
\hline & Viudas (os) & & & & 54 & 16.2 \\
\hline \multicolumn{7}{|c|}{ Nivel de Estudios } \\
\hline & No tienen estudios & & & & 24 & 7.2 \\
\hline & Primaria & & & & 161 & 48.2 \\
\hline & Secundaria & & & & 63 & 18.9 \\
\hline & Bachillerato & & & & 36 & 10.7 \\
\hline & Profesional & & & & 50 & 14.9 \\
\hline \multicolumn{2}{|c|}{ Tiempo de Evolución } & 84 & 66.44 & $4-408$ & & \\
\hline \multicolumn{2}{|c|}{ Nivel de Glucosa en Sangre } & 161 & 71.30 & $63-568$ & & \\
\hline
\end{tabular}

Instituto Mexicano del Seguro Social, IMSS, en la ciudad de Hermosillo, México. En una primera instancia los responsables del presente estudio solicitaron la autorización correspondiente a los directivos del IMSS y a los representantes de las asociaciones para la administración de los instrumentos; una vez obtenida aquella se pidió a cada uno de los participantes su colaboración de manera voluntaria, para lo cual se les describieron los objetivos que se perseguían con el estudio. A quienes aceptaron hacerlo se les hizo entrega del consentimiento informado, que leyeron y firmaron. Por último, se les hizo entrega de los instrumentos y las respectivas hojas de respuesta, ofreciéndoles las indicaciones sobre cómo responder; se les aclaró que en caso de tener alguna duda podían acercarse con los responsables; la administración de los instrumentos se llevó al cabo, para los pacientes del IMSS, tanto en un hospital de zona como en una de las Unidades de Medicina Familiar, mientras que para los pertenecientes a las asociaciones, en dos locales en los que se reúnen periódicamente, en ambos casos en la misma ciudad.

\section{Variables e Instrumentos}

Variables Dependientes. Se consideraron tres comportamientos de adhesión a la terapéutica: ejercicio físico, dieta y consumo de medicamentos en el transcurso de la última semana, mismos que se midieron en un formato tipo Likert con cuatro opciones de respuesta que iban de uno (ningún día de la semana) a cuatro (todos los días de la semana); el valor del alfa de Cronbach para las tres preguntas fue de 0.91 .

Variables Independientes Atributivas. Se consideraron dos variables, una psicológica y otra social: situaciones vinculadas con estrés y apoyo social, respectivamente. La evaluación de cada variable se realizó con base en una escala general y un cuestionario previamente validados en personas con VIH, con los mismos antecedentes socioculturales de los participantes en este estudio. Las situaciones vinculadas con estrés que se tuvieron en cuenta se relacionaban directamente con el tratamiento médico y con la forma en que cada persona con diabetes evaluaba tanto su interacción con el personal de salud como con otras 
personas significativas de su entorno social inmediato: toma de decisiones, tolerancia a la ambigüedad y tolerancia a la frustración. Su medición se realizó con base en una escala (Piña, Valencia, Mungaray \& Corrales, 2006), con la cual se obtuvo un valor del alfa de Cronbach de 0.86 para esta muestra — con valores de $0.92,0.81$ y 0.72 para cada una de las tres situaciones, respectivamente-. Cada pregunta se encontraba definida en una escala de 10 puntos, donde uno representaba una situación que fue valorada como nada amenazante y 10 como demasiado amenazante. Un ejemplo de pregunta sobre toma de decisiones fue el siguiente: "Usted se encuentra recibiendo tratamiento y recibe presiones de personas cercanas para seguir con uno diferente; entonces ello le resulta...". Por su parte, un ejemplo sobre tolerancia a la ambigüedad fue: "A usted se le diagnosticó una enfermedad que no sabe si tendrá cura en el corto o mediano plazos; entonces ello le resulta...". Un ejemplo sobre tolerancia a la frustración fue: "Usted se encuentra recibiendo tratamiento y hace todo lo que se le indica, pero no recibe respuestas favorables o positivas por parte del personal de salud; entonces ello le resulta..."

Por su parte, el apoyo social se midió con el DukeUNC-11, previamente adaptado y validado en México en personas con VIH (Piña, Corrales \& Rivera, 2007), con el cual se obtuvo un valor del coeficiente alfa de Cronbach de 0.95 para esta muestra. Consta de 11 preguntas que se midieron en un formato tipo Likert con cinco opciones de respuesta, que iban de uno - mucho menos de lo que quiero o espero- a cinco - tanto como quiero o espero- - Un ejemplo de pregunta fue el siguiente: "Recibo visitas de mis amigos o familiares".

\section{Análisis Estadístico}

Dadas las características de las variables dependientes e independientes, que fueron definidas con base en escalas ordinales y de intervalo, para los propósitos de los análisis que se detallan más adelante todas las variables se recodificaron en valores "Dummy" de uno y dos. Así, las tres preguntas sobre los comportamientos de adhesión se recodificaron para diferenciar entre no-adherentes (opciones de respuestas uno, dos y tres, que se transformaron en uno) y $100 \%$ adherentes (opción de respuesta cuatro, que se transformó en dos). Algo similar se hizo con los valores de las respuestas a la escala de situaciones vinculadas con estrés y con el cuestionario de apoyo social funcional, para las cuales se tomaron como indicadores los valores de la mediana, en cada caso con el valor menor de la mediana asignándole el uno y al mayor el dos.

En virtud de dichas recodificaciones, en un primer momento se obtuvieron estadísticas descriptivas de las variables de interés. Enseguida, y con el objeto de probar diferencias significativas entre no-adherentes y 100\% adherentes en los comportamientos de adhesión se utilizó el $x^{2}$ de Pearson, aceptándose niveles de significación estadística de $\mathrm{p} \leq 0.005$. Por último, para la identificación de asociaciones entre variables psicológicas y sociales con los comportamientos de adhesión se empleó un análisis de regresión logística multivariada, el cual permite ir evaluando la importancia relativa de cada variable dentro del modelo, incorporando aquellas que son necesarias para la explicación de las variables dependientes, aceptándose niveles de significación estadística de $\mathrm{p} \leq 0.005$; con el propósito de probar la bondad de ajuste de los datos al modelo se utilizó la prueba de Hosmer \& Lemeshow (2001).

\section{Resultados}

Respecto de los comportamientos de adhesión, 104 participantes reportaron haber practicado todos los días de la semana los comportamientos de adhesión relacionados con ejercicio físico (31.1\%), 124 (37.1\%) los relacionados con la dieta y $300(89.8 \%)$ los relacionados con el consumo de medicamentos. Una vez hechas las recodificaciones mencionadas, con el $x^{2}$ de Pearson se encontraron diferencias significativas entre los participantes no-adherentes y $100 \%$ adherentes en los tres comportamientos: ejercicio físico $\left(x^{2}[1]=47.533 ; \mathrm{p}<0.001\right)$, dieta $\left(x^{2}[1]=22.144 ; \mathrm{p}\right.$ $<0.001)$ y consumo de medicamentos $\left(x^{2}[1]=211.844 ; \mathrm{p}\right.$ $<0.001$ ), en los dos primeros haciendo las diferencias los participantes no-adherentes, mientras que en el último los $100 \%$ adherentes.

Por otro lado, los valores de la mediana para las variables psicológicas y sociales fueron los siguientes: de tres para toma de decisiones - de un rango posible de tres a 30 puntos—-, de tres para tolerancia a la ambigüedad —en el mismo rango que la anterior-; de nueve para tolerancia a la frustración — rango posible de seis a 60 puntos—, así como de 55 para apoyo social percibido — rango posible de 11 a 55 puntos-. Esto es, los valores en las tres situaciones vinculadas con estrés se corresponden con los mínimos esperados - a menor puntuación menor es el estrés experimentado en cada situación - en tanto que en apoyo social percibido se corresponden con los máximos esperados, en el sentido de que a mayor puntuación mayor es el apoyo social percibido.

En lo tocante a los análisis de regresión logística multivariada, los resultados se resumen en la Tabla 2. Ejercicio físico se asoció con dos variables: bajo estrés vinculado con toma de decisiones $(\mathrm{RM}=1.14$; IC $95 \%=0.84-2.13) \mathrm{y}$ con tolerancia a la frustración $(\mathrm{RM}=0.42 ; \mathrm{IC} 95 \%=0.25-0.71)$. La prueba ómnibus del modelo conjunto fue significativa $\left(x^{2}[2]=19.286 ; \mathrm{p}<0.001\right)$; por lo que hace a la bondad de ajuste de los datos al modelo ésta fue la óptima, en virtud de que la prueba de Hosmer \& Lemeshow (2001) no fue significativa $\left(x^{2}[2]=0.488 ; \mathrm{p}=0.485\right)$.

En la misma Tabla 2 se resumen los resultados para dieta, comportamiento que se asoció con dos variables: bajo estrés vinculado con tolerancia a la frustración $(\mathrm{RM}=0.50$; 
IC $95 \%=0.31-0.80)$ y con apoyo social $(\mathrm{RM}=0.47$; IC $95 \%=0.28-0.76$ ). La prueba ómnibus del modelo conjunto también fue significativa $\left(x^{2}[2]=9.911 ; \mathrm{p}<0.005\right)$, en tanto que la bondad de ajuste de los datos al modelo también fue la óptima, pues la prueba de Hosmer \& Lemeshow (2001) no fue significativa $\left(x^{2}[2]=5.283 ; \mathrm{p}=0.071\right)$.

Por último, el análisis para consumo de medicamentos no aportó ninguna asociación, lo que se confirma con la prueba ómnibus del modelo conjunto, pues ésta no fue significativa $\left(x^{2}[4]=4.891 ; \mathrm{p}=0.087\right)$. En razón de este resultado y partiendo de la hipótesis de que el problema tendría eventualmente que ver con diferencias entre los derechohabientes del IMSS y quienes pertenecen a las asociaciones, se realizó un siguiente análisis considerando ambos grupos como variable criterio.

El análisis en cuestión demostró que nuestra hipótesis era la correcta, pues para el grupo de participantes derechohabientes del IMSS se encontraron dos asociaciones para el comportamiento consumo de medicamentos (Tabla 3): con bajos niveles de estrés vinculado con toma de decisiones $(\mathrm{RM}=1.90 ;$ IC $95 \%=1.23-4.04)$ y con apoyo social $(\mathrm{RM}=1.00 ; \mathrm{IC} 95 \%=0.83-2.21)$. La prueba ómnibus del modelo fue significativa $\left(x^{2}[2]=21.156 ; \mathrm{p}<0.001\right)$ y la bondad de ajuste de los datos fue la óptima, toda vez que la prueba de Hosmer \& Lemeshow (2001) no fue significativa $\left(x^{2}[2]=0.057 ; \mathrm{p}=0.903\right)$. Respecto de los participantes pertenecientes a las asociaciones de diabéticos no se encontró ninguna asociación, que se confirma con la prueba ómnibus del modelo, en tanto que ésta no fue significativa $\left(x^{2}[4]=1.032 ; \mathrm{p}=0.120\right)$.

\section{Discusión}

En una revisión sistemática de 23 estudios relacionados con la adhesión a la terapéutica en personas con diagnóstico de diabetes tipo 2, Cramer (2004) encontró en 15 estudios retrospectivos que los porcentajes de adhesión con hipoglucemiantes orales e inyección de insulina oscilaron entre 36 y $93 \%$, considerando a muestras de personas mayores de 50 años, edad parecida a la de los participantes del presente estudio, cuya mediana fue de 55 años.

Lo anterior es especialmente interesante, si para dicho propósito se comparan los hallazgos reportados por el autor con los obtenidos por otros tantos en nuestro país. Por ejemplo, en un estudio conducido por Hernández-Ronquillo, Téllez-Zenteno, Garduño-Espinosa \& González-Aceves (2003) se encontró que los porcentajes de adhesión para diferentes comportamientos fueron bajos en ejercicio físico $(15 \%)$ y adopción de una dieta (38\%), mientras que para consumo de medicamentos e inyección de insulina fueron altos ( 83 y $87 \%$, respectivamente), relativamente similares a los obtenidos aquí, con excepción del primero de ellos.

En otro se señala que el porcentaje de adhesión para consumo de medicamentos fue de 54\% (Durán-Varela, Rivera-Chavira \& Franco-Gallegos, 2001), poco más de 30 puntos porcentuales por debajo del encontrado en nuestro estudio. En un tercero se encontraron, para adopción de una dieta balanceada porcentajes entre 17.7 y $63.3 \%$, mientras que para consumo de medicamentos entre 11 y $91.6 \%$ (Garay-Sevilla, et al., 1995). Finalmente, Samaniego \& Álvarez (2006) obtuvieron porcentajes elevados en los comportamientos adopción de una dieta balanceada (67\%) y realizar ejercicio físico (68.1\%), aunque bajos en consumo de medicamentos (55\%), siendo más notorias las diferencias respecto de las reportadas aquí.

Aún cuando se trata de estudios que sin lugar a dudas aportan información valiosa en términos de que fue posible conocer los porcentajes con que se practican los diferentes comportamientos de adhesión por parte de las personas con diagnostico de diabetes tipo 2, presentan el inconveniente de carecer de sustento en modelos teóricos que eventualmente nos permitan procurar explicaciones mejor fundadas sobre las variables psicológicas y sociales que facilitan o impiden

Tabla 2. Análisis de regresión logística multivariado de comportamientos de adhesión en personas con diabetes tipo 2.

\begin{tabular}{lcccc}
\hline Comportamientos y Variables & $\beta$ & EE & Wald & $\mathrm{p}$ \\
\hline Ejercicio fisico: & & & & $<0.01$ \\
Toma de decisiones & -0.890 & 10.348 & 6.552 & $<0.001$ \\
Tolerancia a la frustración & -1.850 & 0.262 & 10.561 & $<0.005$ \\
Constante & 0.519 & 0.164 & 3.031 & \\
& & & & $<0.005$ \\
Dieta: & & & & $<0.005$ \\
Tolerancia a la frustración & -0.684 & 0.237 & 8.320 & 0.623 \\
Apoyo social & 0.756 & 0.252 & 9.036 & 0.241 \\
Constante & 0.085 & 0.174 & & \\
\hline
\end{tabular}


Tabla 3. Análisis de regresión logística multivariado del comportamiento consumo de medicamentos para los participantes del IMSS.

\begin{tabular}{lcccc}
\hline Comportamientos y variables & $\beta$ & EE & Wald & \\
\hline Consumo de medicamentos: & & & & \\
Toma de decisiones & -3.212 & 0.215 & 15.192 & $<0.001$ \\
Apoyo social & 2.147 & 0.431 & 12.874 & $<0.001$ \\
Constante & 2.228 & 0.487 & 13.101 & $<0.001$ \\
\hline
\end{tabular}

la práctica eficiente de los mencionados comportamientos. En ese contexto, lo que hace interesante a nuestro estudio no sólo tiene que ver con el hecho de que se justificó en un modelo teórico de reciente creación, sino las variables psicológicas y sociales que se asociaron con los tres comportamientos de adhesión: dos situaciones vinculadas con estrés y apoyo social percibido; ahora bien, ¿cómo es que podemos explicar el papel de esas variables desde un punto de vista teórico?

En el caso de la situación vinculada con toma de decisiones, hay que tener en cuenta que se trata de una en la que es clave el papel de las señales o eventos de estímulo previo a una interacción, en tanto que en el caso de la situación vinculada con tolerancia a la frustración lo es el papel de las consecuencias de estímulo al comportarse. Constituyen el referente inmediato para identificar cómo cada persona ha aprendido a entrar en contacto con determinadas situaciones en las que no se prescribe de antemano criterio alguno sobre qué hacer o cómo comportarse, y en tal sentido permiten identificar las funciones particulares que describen a cada persona siendo diferente del resto (Ribes, 1990).

Toda vez que cuando se habla de situaciones interactivas en éstas se encuentran implicados objetos de estímulo, acontecimientos u otras personas a los/las que se responde, el papel de cada uno podrá adquirir menor o mayor relevancia para la forma en que cada persona se comporte en las diferentes situaciones. Así, por ejemplo, desde la óptica del modelo aquí utilizado, tomar decisiones constituye un proceso eminentemente psicológico en el que las personas, al enfrentar situaciones tanto de la vida cotidiana como otras relacionadas con la enfermedad y el tratamiento, deben ser capaces de identificar el tipo de demanda a la que hay que responder - por ejemplo, realizar ejercicio físico dosificado todos los días de la semana (Makoul \& Clayman, 2006; Weymiller et al., 2007): se toman decisiones y se actúa de manera congruente, esto es, realizando ejercicio físico cada día de la semana. Si llegado el momento las personas con diabetes identifican o perciben correctamente la demanda y ésta no representa una "amenaza", técnicamente hablando estaríamos haciendo referencia a situaciones que no favorecerían el desarrollo del fenómeno conocido como estrés (Piña \& Torres, 2008). Por tanto, se trataría de situaciones que eventualmente facilitarían un mejor desempeño competencial y su actualización en la forma de comportamientos de adhesión - hay que recordar que en su conjunto los par- ticipantes de esta muestra obtuvieron la puntuación mínima esperada de tres puntos en aquella variable.

Por lo que hace al papel de las situaciones vinculadas con tolerancia a la frustración, en éstas son claves las consecuencias al responder. Si se considera que la puntuación mediana ascendió a nueve puntos - de un rango posible de seis a 60 puntos-, estamos ante a un fenómeno que sugiere una baja valoración de estrés cuando los participantes enfrentan situaciones que presuponen practicar los comportamientos de adhesión. Esto último ocurrió al margen de que las consecuencias de estímulo que se obtuvieran fueran menores que las esperadas, se demoraran, se les retiraran sin razón o que requirieran de una ejecución mayor para obtenerlas. Empero, esto último es particularmente interesante, pues pareciera que existe una contradicción entre lo que se plantea en la mencionada situación vinculada con estrés y los porcentajes que se obtuvieron en los dos comportamientos con los que se asoció, que se caracterizaron por obtener justo los porcentajes más bajos; nos referimos a realizar ejercicio físico $(31.1 \%)$ y adoptar una dieta $(37.1 \%)$.

Es posible, a modo de hipótesis — que deberá por supuesto someterse a las pruebas correspondientes-, que se trate de un asunto en el que, por las propias características de ambos comportamientos, gradualmente demanden una ejecución mayor (Hodge, English, O’Dea \& Giles, 2007; Martinus, Corban, Wackerhage, Atkins \& Singh, 2006). En efecto, en términos prácticos no es lo mismo dedicar cierto tiempo al día a realizar ejercicio físico y adoptar una dieta, que por ejemplo el que se destina a consumir los medicamentos, en particular cuando se está trabajando con niños o adultos mayores. Constituyen dos grupos que, por su propia condición, se encuentran expuestos a exigencias a las que no pueden responder como tales, en la medida en que se tienen que ajustar diferencialmente a criterios impuestos por otras personas de significativas de su entorno social inmediato (Huang, Gorawara \& Chin, 2005; Martin, Riquelme \& Lechuga, 2007).

En tal sentido, como plantearan tiempo atrás Cofer \& Appley (1964/1982), los fenómenos de estrés y frustración no pueden concebirse siendo independientes, de forma tal que las personas con diabetes pareciera que van aprendiendo a regular su propio comportamiento en función de ciertas consecuencias de estímulo, como eventualmente podría ser el conocimiento que se tiene del estado de salud, por la manifestación o ausencia de signos y síntomas relacionadas con otras afecciones en el planos biológico, así como por la 
manera en que interactúan con otras personas significativas de su entorno social inmediato (Ponce, Velázquez, Márquez, López \& Bellido, 2009; Rosland et al., 2008).

De ahí que, sobre esto último, llame poderosamente nuestra atención el que la variable apoyo social se asociara tanto con adoptar una dieta como con consumir los medicamentos. Es decir, si para los dos comportamientos previamente analizados se comentó que hay una relación especial entre las señales de estímulo, la práctica de los comportamientos y las consecuencias al responder, la pregunta que hay que hacer es cuál es el papel que juega el apoyo social a lo largo del proceso de interacción. Una respuesta inicial nos tendría que llevar a plantear que cada comportamiento, en virtud de que adquiere funcionalidad propia, se encuentra regulado por la influencia diferencial de variables psicológicas y sociales de diversos tipos, como las aquí analizadas, o bien otras que no se consideraron en esta ocasión, como serían los casos de los motivos y las competencias conductuales.

En segundo lugar, habría que entender también que las consecuencias que generan los diferentes comportamientos no son necesariamente equivalentes en cada caso, y en ocasiones pueden ser dispensadas por el personal de salud $y$ en otras por personas significativas del entorno social inmediato (McBride, Owens, Brody, Black, Wilert \& Brown, 2003; Tang et al., 2008). Por lo tanto, si el apoyo social percibido alcanzó la puntuación mediana más alta esperada, equivalente a los 55 puntos, todo parece indicar que para los comportamientos con los que se asoció es fundamental el apoyo que brindan a los participantes esas otras personas significativas, trátese de la pareja, de los familiares, de los amigos e inclusive del propio personal de salud.

En síntesis, la práctica consistente y eficiente de tres comportamientos de adhesión en personas con diabetes tipo 2 se vio afectada de manera diferencial por situaciones vinculadas con estrés que se relacionan tanto con las señales o eventos de estimulo previo a una interacción como a las consecuencias al responder; en la misma dirección se plantea el papel del apoyo social percibido y el rol específico de otras personas significativas del entorno social inmediato. Toda vez que esas variables afectan diferencialmente la práctica de los mencionados comportamientos, previo al diseño de programas de intervención dirigidos a promover éstos, es preciso identificar qué y cómo las variables psicológicas y sociales se asocian con su ejercicio instrumental.

Si bien es cierto que los resultados de este estudio arrojan información valiosa sobre las maneras en que un conjunto de variables psicológicas y sociales se asociaron con tres comportamientos de adhesión en personas con diabetes tipo 2, es necesario reconocer algunas limitaciones. Por un lado, el tamaño de la muestra, que se circunscribió a personas que eran atendidas en un hospital y una unidad de medicina familiar de una institución de seguridad social, así como a otras pertenecientes a dos asociaciones civiles de la localidad. Por otro lado, son de llamar la atención los valores de las razones de momios y sus correspondientes intervalos de confianza obtenidos en el análisis de regresión para los comportamientos ejercicio físico (parcialmente) y dieta (en su conjunto), pues respecto de los primeros se ubicaron en esos casos por debajo de 1.0, con independencia de que la prueba ómnibus resultara significativa y la de Hosmer \& Lemeshow no. Asimismo, a pesar de se encontró un peso importante de dos situaciones vinculadas con estrés y del apoyo social sobre los comportamientos de adhesión, no fue posible incorporar otras variables, como por ejemplo la evaluación sistemática de los motivos, el nivel competencial pasado y otros comportamientos asociados a la enfermedad, caso de la depresión. Asimismo, el que por razones de tipo económico y administrativas no se hayan considerado los niveles de hemoglobina glucosilada y la identificación puntual de complicaciones clínicas, impuso limitaciones que nos impidieron realizar otros análisis, que en el caso de las personas con diabetes son fundamentales.

Por lo anterior, será necesario que en próximos estudios se incluyan muestras más amplias de personas con diagnóstico de diabetes tipo 2, que se encuentran recibiendo atención especializada en otras dos instituciones de seguridad social de la localidad. En el mismo tenor será necesario replantear el análisis estadístico, intentando modelar con ecuaciones estructurales cómo es que interactúan las variables de proceso y resultado del modelo.

Finalmente, al tratarse de un segundo estudio que se justificó en el modelo psicológico para la investigación de los comportamientos de adhesión en personas con diabetes tipo 2, debe entenderse como una prioridad la necesidad de ampliar y mejorar nuestro conocimiento respecto de cómo, por qué y bajo qué circunstancias las personas practican o no de manera consistente y eficiente los comportamientos de adhesión prescritos por el personal de salud. Esto último es clave para estar en una mejor posición a efecto de diseñar, instrumentar y evaluar programas de intervención de naturaleza interdisciplinaria, tendientes a promover la práctica de los mencionados comportamientos en los términos expuestos.

\section{Referencias}

Babwah, F., Baksh, S., Blake, L., Cupid-Thuesday, J., Hosein, I., Sookhai, A., et al. (2006). The role of gender in compliance and attendance at an outclinic for type 2 diabetes mellitus in Trinidad. Revista Panamericana de Salud Pública, 19, 79-84.

Barceló, A., Aedo, C., Rajpathak, S., \& Robles, S. (2003). The cost of diabetes in Latin America and the Caribbean. Bulletin of the World Health Organization, 81, 19-27.

Brownson, C.A. \& Heisler, M. (2009). The role of peer support in diabetes care and self-management. The Patient-Centered Outcomes Research, $2,5-17$

Cofer, C.N. \& Appley, M.H. (1964/1982). Psicología de la motivación. Teoría e investigación. México: Editorial Trillas. 
Córdova-Villalobos, J.A., Barriguete-Meléndez, J.A., Lara-Esqueda, A., Barquera, S., Rosas-Peralta, M., Hernández-Ávila, M., et al. (2008). Las enfermedades crónicas no transmisibles en México: sinopsis epidemiológica y prevención integral. Salud Pública de México, 50, 419-427.

Cramer, J.A. (2004). A systematic review of adherence with medications for diabetes. Diabetes Care, 27, 1218-1224.

Durán-Varela, B.R., Rivera-Chavira, B., \& Franco-Gallegos, E. (2001). Apego al tratamiento farmacológico en pacientes con diagnóstico de diabetes mellitus tipo 2. Salud Pública de México, 43, 233-236.

Fisher, L., Skaff, M.M., Mullan, J.T., Arean, P., Mohr, D., Masharani, V., et al. (2007). Clinical depression versus distress among patients with type 2 diabetes: not just a question of semantics. Diabetes Care, 30, 542-547.

Garay-Sevilla, M.E., Nava, L.E., Malacara, J.M., Huerta, R., Díaz de León, J., Mena, A., et al. (1995). Adherence to treatment and social support in patients with non-insulin dependent diabetes mellitus. Journal of Diabetes and its Complications, 9, 81-86.

Hernández-Ronquillo, L., Téllez-Zenteno, J.F., Garduño-Espinosa, J., \& González-Aceves, E. (2003). Factors associated with therapy noncompliance in type-2 diabetes patients. Salud Pública de México, 45 , 191-197.

Hodge, A.M., English, D.R., O’Dea, K., \& Giles, G.G. (2007). Dietary patterns and diabetes incidence in the Melbourne collaborative cohort study. American Journal of Epidemiology, 165, 603-610.

Hosmer, D.W., \& Lemeshow, M.P. (2001). Applied logistic regression. New York: John Wiley \& Sons.

Huang, E.S., Gorawara, R., \& Chin, M.H. (2005). Self-reported goals of older patients with type 2 diabetes mellitus. Journal of the American Geriatrics Society, 53, 306-311.

Makoul, G., \& Clayman, M.L. (2006). An integrative model of shared decision making in medical encounters. Patient Education and Counseling, 60, 301-312.

Martín, M., Riquelme, A., \& Lechuga, M. (2007). Factores psicológicos asociados al reporte de cumplimiento terapéutico en pacientes con múltiples enfermedades somáticas crónicas. Psicología y Salud, 17, 63-68.

Martinus, R., Corban, R., Wackerhage, H., Atkins, S., \& Singh, J. (2006). Effect of psychological intervention on exercise adherence in type 2 diabetic subjects. Annals of the New York Academy of Sciences, 1084, 350-360.

McBride, V., Owens, M.D., Brody, G.H., Black, A.R., Wilert, A.S., \& Brown, A.C. (2003). Factors and processes associated with physical and psychological health of African-American mothers with type 2 diabetes: A heuristic model. Diabetes Spectrum, 16, 166-171.

O'Hea, E.L., Moon, S., Grothe, K.B., Boudreaux, E., Bondelos, J.S., Wallston, K., et al. (2009). The interaction of locus of control, selfefficacy, and outcome expectancy in relation to HbAlc in medically undeserved individuals with type 2 diabetes. Journal of Behavioral Medicine, 32, 106-117.
Piña, J.A. (2009). Motivación en psicología y salud: motivación no es sinónimo de intención, actitud o percepción de riesgo. DiversitasPerspectivas en Psicología, 5, 27-35.

Piña, J.A., Corrales, A.E., \& Rivera, B.M (2007). Validación del cuestionario de apoyo social funcional (Duke-UNC-11) en personas seropositivas al VIH del noroeste de México. Ciencia y Enfermería, XIII, 53-63.

Piña, J.A., \& Sánchez-Sosa, J.J. (2007). Modelo psicológico para la investigación de los comportamientos de adhesión terapéutica en personas con VIH. Universitas Psychologica, 6, 399-407.

Piña, J.A., \& Torres, A.M. (2008). Stress-related situations as predictors of adherence-to-medication behavior among women with type 2 diabetes. International Journal of Hispanic Psychology, 1, 75-83.

Piña, J.A., Valencia, M.A., Mungaray, K., \& Corrales, A.E. (2006). Validación de una escala breve que mide situaciones vinculadas con estrés en personas con VIH. Terapia Psicológica, 24, 15-21.

Piña, J.A., Ybarra, J.L., Alcalá, I.G. \& Samaniego, R.A. (en prensa). Psicología y salud (I): la importancia de llamarse modelo y apellidarse teóricoconceptual. Revista Mexicana de Investigación en Psicología, 2.

Ponce, J.M., Velázquez, A., Márquez, E., López, L., \& Bellido, M.L. (2009). Influencia del apoyo social en el control de las personas con diabetes. Índex de Enfermería, 18, 224-228.

Rae, R. (2006). Practical review of oral antihyperglycemic agents for type 2 diabetes mellitus. The Diabetes Educator, 32, 869-876.

Ribes, E. (1990). Psicología y salud: un análisis conceptual. Barcelona: Martínez Roca.

Ribes, E. (2005). ¿Qué es lo que se debe medir en psicología? El asunto de las diferencias individuales. Acta Comportamentalia, 13, 37-52.

Rosland, A-M., Kieffer, E., Israel, B., Cofield, M., Palmisano, G., Sinco, B., et al. (2008). When is social support important? The association of family support and professional support with specific diabetes self-management behaviors. Journal of General Internal Medicine, 23, 1992-1999.

Samaniego, R.A., \& Álvarez, J. (2006). Control de la enfermedad en pacientes con diabetes mellitus tipo 2: una muestra regiomontana. Psicología y Salud, 16, 63-70.

Secretaría de Salud (2006). Principales causas de morbilidad en México en el periodo 2000-2004. México: Autor.

Tang, T.S., Brown, M.B., Funnell, MM., \& Anderson, R.M. (2008). Social support, quality of life, and self-care behaviors among AfricanAmericans with type 2 diabetes. Diabetes Spectrum, 34, 266-276.

Weymiller, A.J., Montori, V.M., Jones, L.A., Gafni, A., Guyatt, G.H., Bryant, S.C., et al. (2007). Helping patients with type 2 diabetes mellitus make treatment decisions. Archives of Internal Medicine, 167, 1976-1082. 